\title{
Declining Inpatient Mortality Despite Increasing Thirty-Day Readmissions of Alcoholic Hepatitis in the United States From 2010 to 2018
}

\author{
Dushyant Singh Dahiya ${ }^{a}$, f $@$, Asim Kichloo, ${ }^{a}$, Jagmeet Singh ${ }^{c}$, Gurdeep Singh ${ }^{d}$, \\ Farah Wani ${ }^{b}$, Hafeez Shaka ${ }^{\mathrm{e}}$
}

\begin{abstract}
Background: Alcoholic hepatitis ( $\mathrm{AH}$ ) readmissions are commonly secondary to relapse to alcohol use after discharge from the hospital.

Methods: This retrospective interrupted trend study analyzed the National Readmissions Database (NRD) from 2010 to 2018 to identify 30-day readmissions of $\mathrm{AH}$ using the International Classification of Diseases (ICD)-9 and ICD-10 codes (571.1 and K70.1). Individuals $<18$ years, elective and traumatic readmissions were excluded. The biodemographic characteristics and hospitalization trends were highlighted over an 8-year time frame. A multivariate regression analysis was used to calculate the risk-adjusted odds of trends for all-cause 30-day readmissions, AH-specific readmissions, inpatient mortality, mean length of stay (LOS), and mean total hospital charge (THC) after adjusting for age, gender, grouped Charlson Comorbidity Index (CCI), type of insurance, mean household income, and hospital characteristics. P-values $\leq 0.05$ were considered statistically significant.
\end{abstract}

Results: We noted an increasing trend for total 30-day readmissions of AH from 1,839 in 2010 to 3,784 in 2018 (P-trend < 0.001). Males made up a majority of the population; however, gender distribution was not statistically significant. Additionally, 30-day AH readmissions had an increasing comorbidity burden with time. The 30-day all-cause readmission rate increased from $18.8 \%$ in 2010 to $24.4 \%$ in 2018 and $\mathrm{AH}-$ specific readmission rate from $2.9 \%$ in 2010 to $3.9 \%$ in 2018 without a statistically significant trend. However, we noted a declining risk-adjusted trend of inpatient mortality for 30-day readmissions of $\mathrm{AH}$ from $8.7 \%$ in 2010 to $7.4 \%$ in 2018 (P-trend $=0.022$ ). Furthermore, the total LOS attributable to 30-day readmissions of AH increased by $132.5 \%$ from 11,275 days in 2010 to 26,220 days in 2018 and the attributable

Manuscript submitted October 13, 2021, accepted November 11, 2021

Published online November 29, 2021

${ }^{a}$ Central Michigan University College of Medicine, Saginaw, MI, US

bSamaritan Medical Center, Watertown, NY, US

${ }^{\mathrm{c}}$ Guthrie Robert Packer Hospital, Sayre, PA, US

dOur Lady of Lourdes Memorial Hospital, Binghamton, NY, US

e John H. Stroger, Jr. Hospital of Cook County, Chicago, IL, US

${ }^{f}$ Corresponding Author: Dushyant Singh Dahiya, Central Michigan University

College of Medicine, 1000 Houghton Ave., Saginaw, MI 48603, US.

Email: dush.dahiya@gmail.com

doi: https://doi.org/10.14740/gr1473
THC increased by $160.9 \%$ to over $\$ 67$ million in 2018 .

Conclusions: For 30-day AH readmissions, inpatient mortality declined to $7.4 \%$ in 2018 , while the total number of hospitalizations, LOS and THC increased from 2010 to 2018.

Keywords: Alcoholic hepatitis; Hepatitis; Mortality; Outcomes; Trends

\section{Introduction}

Excess consumption of alcohol places an enormous socioeconomic burden on both individuals and healthcare systems across the globe [1]. From a clinical perspective, alcohol abuse is known to lead to multi-systemic cellular injury; however, the liver sustains the highest degree of cellular damage as it is the primary site of ethanol metabolism [2]. Alcohol liver disease (ALD) encompasses a wide spectrum of clinical conditions ranging from reversable fatty changes of the liver to irreversible cirrhosis and in some cases progression to hepatocellular carcinoma (HCC) [3]. Of the ALDs, alcoholic hepatitis (AH) is a distinct, acute, inflammatory liver disease secondary to chronic alcohol abuse associated with a poor prognosis [4]. In literature, $\mathrm{AH}$ has been associated with mortality rates as high as $40 \%$ within a 180 -day period along with a nine times higher risk of progression to liver cirrhosis as compared to patients with only hepatic steatosis [5]. Patients with AH are at increased risk of subsequent hospitalizations and mortality due to sudden hepatic decompensation or relapse to alcohol use post hospital discharge [6]. However, there is significant paucity of data on early (within 30 days of hospital discharge) readmissions of AH with only a few studies available in literature. Therefore, this study was designed to identify hospitalizations characteristics, trends of readmissions and outcomes for 30-day readmissions of $\mathrm{AH}$.

\section{Materials and Methods}

\section{Design and data source}

The data analyzed for this retrospective interrupted trend study was extracted from the Nationwide Readmissions Database (NRD) which is the largest, publicly available readmission database in the 
US drawn from the Agency for Healthcare Research and Quality (AHRQ) Healthcare Cost and Utilization Project (HCUP) State Inpatient Databases [7]. For each calendar year, NRD contains discharge information from geographically dispersed and diverse states. It stores both patient-level and hospital-level information, and the hospitals are stratified according to ownership control, number of beds, teaching status, and metropolitan/non-metropolitan location. The NRD allows for weighted analysis to obtain $100 \%$ of the US readmissions within a given year.

\section{Study population}

This study analyzed hospitalizations from the NRD for the years 2010, 2012, 2014, 2016 and 2018 with AH as the cause for index admission using the International Classification of Diseases (ICD)-9 and ICD-10 codes (571.1 and K70.1). Using unique hospitalization identifiers, index hospitalizations were identified and one subsequent hospitalization within 30 days for $\mathrm{AH}$ was tagged as a readmission. Individuals $<18$ years, traumatic and elective hospitalizations were excluded from the study.

\section{Statistical analysis and outcome measures}

The data were analyzed using Stata ${ }^{\circledR}$ Version 16 software (StataCorp, Texas, US). All analyses were performed using weighted samples for national estimates in adjunct with HCUP regulations for the utilization of the NRD. The comorbidity burden was assessed using Sundararajan's adaptation of the modified Deyo's Charlson Comorbidity Index (CCI) [8]. A multivariate regression analysis was used to calculate the risk-adjusted odds of trend for all-cause 30-day readmissions, AH-specific readmissions, inpatient mortality, length of stay (LOS), and total hospital charge (THC) after adjusting for age, gender, grouped CCI, type of insurance, mean household income, and hospital characteristics. The THC was obtained using the HCUP Cost-to-Charge Ratio files and adjusted for inflation using the Medical Expenditure Panel Survey index for hospital care, with 2018 as the reference point $[9,10]$. All P-values were 2 sided, with 0.05 set as the threshold for statistical significance.

\section{Ethical considerations}

The NRD lacks specific patient and hospital identifiers. Therefore, this study was exempt from Institutional Review Board (IRB) approval as per guidelines put forth by our institutional IRB for NRD database studies.

\section{Results}

\section{Hospitalization characteristics for 30-day AH readmissions}

There was a rising trend for the total number of 30-day readmissions of AH from 1,839 in 2010 to 3,784 in 2018 (P- trend $<0.001)$. The mean age was noted to be around 46 years (Table 1). Although a higher proportion of the readmissions were observed for males, there was no statistically significant trend for gender. Additionally, 30-day readmissions for $\mathrm{AH}$ were observed to have an increasing comorbidity burden over time (Table 1). From a hospital perspective, large bed-sized and metropolitan hospitals had the highest proportion of 30day $\mathrm{AH}$ readmissions. However, an increasing trend for 30-day readmissions of $\mathrm{AH}$ was noted only for metropolitan teaching hospitals with an increase from $50.3 \%$ in 2010 to $75.1 \%$ in 2018 (P-trend < 0.001).

\section{Readmission trends for 30-day AH readmissions}

The 30-day all-cause readmission rate of $\mathrm{AH}$ increased from $18.8 \%$ in 2010 to $24.4 \%$ in 2018 (P-trend $=0.107$ ) and the 30 day AH-specific readmission rate increased from $2.9 \%$ in 2010 to $3.9 \%$ in 2018 (P-trend $=0.546$ ) without a statistical trend after adjusting for patient-specific and hospital-specific factors (Table 2). Additionally, the 30-day AH readmission proportion increased from $18.6 \%$ in 2010 to $20 \%$ in 2018 without a statistical trend $(\mathrm{P}$-trend $=0.402)$. However, inpatient mortality had a declining risk-adjusted trend from $8.7 \%$ in 2010 to $7.4 \%$ in $2018($ P-trend $=0.022)($ Table 2$)$.

\section{Impact of 30-day $\mathrm{AH}$ readmissions on the United States healthcare system}

From 2010 to 2018, the total LOS attributable to 30-day AH readmissions increased by $132.5 \%$ from 11,275 days in 2010 to 26,220 days in 2018 . The mean LOS per readmission increased from 6.1 days in 2010 to 6.7 days in 2018 (P-trend $=$ 0.822 ) without a statistical trend (Table 2). Furthermore, the THC attributable to 30-day AH readmissions increased by $160.9 \%$ to over $\$ 67$ million in 2018 while the mean THC per admission increased from $\$ 14,285$ in 2010 to $\$ 17,227$ in 2018 $(\mathrm{P}$-trend $=0.306)$ without a statistically significant trend. Medicaid was the largest insurer for the study period, followed by private insurance and Medicare.

\section{Discussion}

This study evaluates the trends of readmissions and outcomes for 30-day readmissions of AH from 2010 to 2018. We noted an increasing trend for the total number of 30-day readmissions of AH from 1,839 in 2010 to 3,784 in 2018 (P-trend < 0.001 ) with metropolitan teaching hospitals accommodating the highest readmissions. The 30-day all-cause readmission rates, 30-day $\mathrm{AH}$-specific readmission rate and 30-day $\mathrm{AH}$ readmission proportion increased from 2010 to 2018 without a statistical trend. However, inpatient mortality had a declining risk-adjusted trend from 8.7\% in 2010 to $7.4 \%$ in 2018 (Ptrend $=0.022)($ Fig. 1). Furthermore, the total LOS and THC attributable to 30-day $\mathrm{AH}$ readmissions increased by $132.5 \%$ and $160.9 \%$, respectively. 
Table 1. Demographic and Hospitalization Characteristics for 30-Day Readmissions of Alcoholic Hepatitis

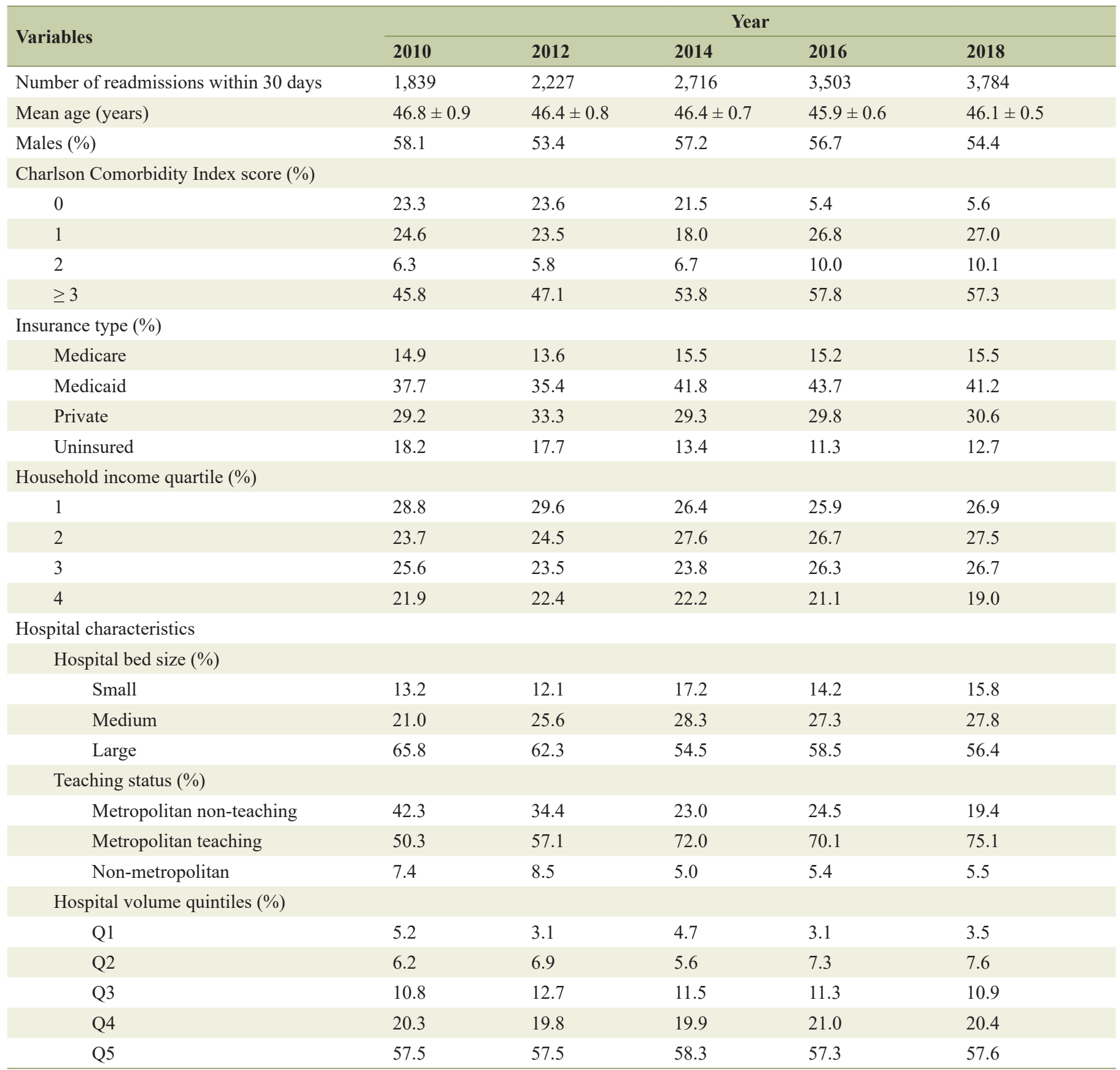

AH negatively impacts the patient's quality of life and places significant burden on the healthcare system. Furthermore, in patients who survive the first inpatient admission for $\mathrm{AH}$, it is paramount to prevent relapse to alcohol use as the long-tern survival depends on abstinence from alcohol rather than the severity of the disease [11]. In current literature, the relapse rates have been reported to be as high as 25\% at 1 year and $60 \%$ at 5 years $[11,12]$. Therefore, it is essential to estimate the magnitude of 30-day readmissions of $\mathrm{AH}$ and its impact on individuals and the healthcare system. In the US from 2006 to 2014, alcohol-related emergency department (ED) visits increased by $47 \%$ accounting for an annual average increase of 210,000 [13]. A retrospective study from 2010 to 2014 noted that 30 -day and 90-day readmissions for $\mathrm{AH}$ related hospitalizations were $22.8 \%$ and $36.6 \%$, respectively in the US with rates that were statistically unchanged for the study period [14]. Furthermore, from a mortality perspective, a systematic review by Hughes et al reported that the overall mortality of AH from 1971 to 2016 was $26 \%$ at 28 days, $29 \%$ at 90 days and $44 \%$ at 180 days after hospitalization and has 
Table 2. Trends of Readmission Rates, Adverse Outcomes, and Healthcare Utilization for 30-Day Readmissions of Alcoholic Hepatitis

\begin{tabular}{|c|c|c|c|c|c|c|}
\hline \multirow{2}{*}{ Outcome } & \multicolumn{5}{|c|}{ Year } & \multirow{2}{*}{ P-trend } \\
\hline & 2010 & 2012 & 2014 & 2016 & 2018 & \\
\hline All-cause readmission rate $(\%)$ & 18.8 & 19.9 & 21.3 & 25.9 & 24.4 & 0.107 \\
\hline Alcoholic hepatitis readmission proportion (\%) & 18.6 & 18.0 & 16.6 & 18.1 & 20.0 & 0.402 \\
\hline Inpatient mortality $(\%)$ & 8.7 & 8.5 & 8.5 & 9.1 & 7.4 & 0.022 \\
\hline
\end{tabular}

not improved with time [15].

In this study, we noted an increase in the total number of readmissions within 30 days following index hospitalization of $\mathrm{AH}$. This rising trend was expected as there is an increased consumption and dependence on alcohol use in the US population. Additionally, relapse to alcohol use, unwillingness to participate in an alcohol rehabilitation program post-discharge and sudden hepatic decompensation in these patients may also have a role to play. Population-based studies conducted in the US have reported the highest prevalence of ALD in the 45 - 64 age group with a mean age of 53 years [16]. A male predominance has also been reported [16]. Our study echoed these findings as the mean age for 30-day $\mathrm{AH}$ readmissions was noted to be approximately 46 years and a male predominance without a statistical trend for gender (Table 1). Furthermore, we noted an increase in the 30-day all-cause readmission rate, 30-day AH-specific readmission rate and 30-day AH readmis- sion proportion without a statistical trend. These findings were in line with current literature in terms of similar proportions for 30-day AH readmission and statistically unchanged readmission rates for the study period [14]. However, we observed a declining risk-adjusted trend of inpatient mortality for 30-day AH readmissions from $8.7 \%$ in 2010 to $7.4 \%$ in 2018 . These findings contradict prior studies which reported higher 30-day mortality with no improvement over time [15]. Although the exact reason for this downtrend in mortality, despite increasing trend of 30-day readmissions, is currently unknown, we hypothesize that it may in part reflect better management strategies and early access of subspeciality care for these patients. Nonetheless, we advocate for the need of additional large, prospective multi-center studies to further investigate these trends of inpatient mortality.

Moreover, with an increase in the overall 30-day readmissions of $\mathrm{AH}$, the financial burden on the US healthcare system

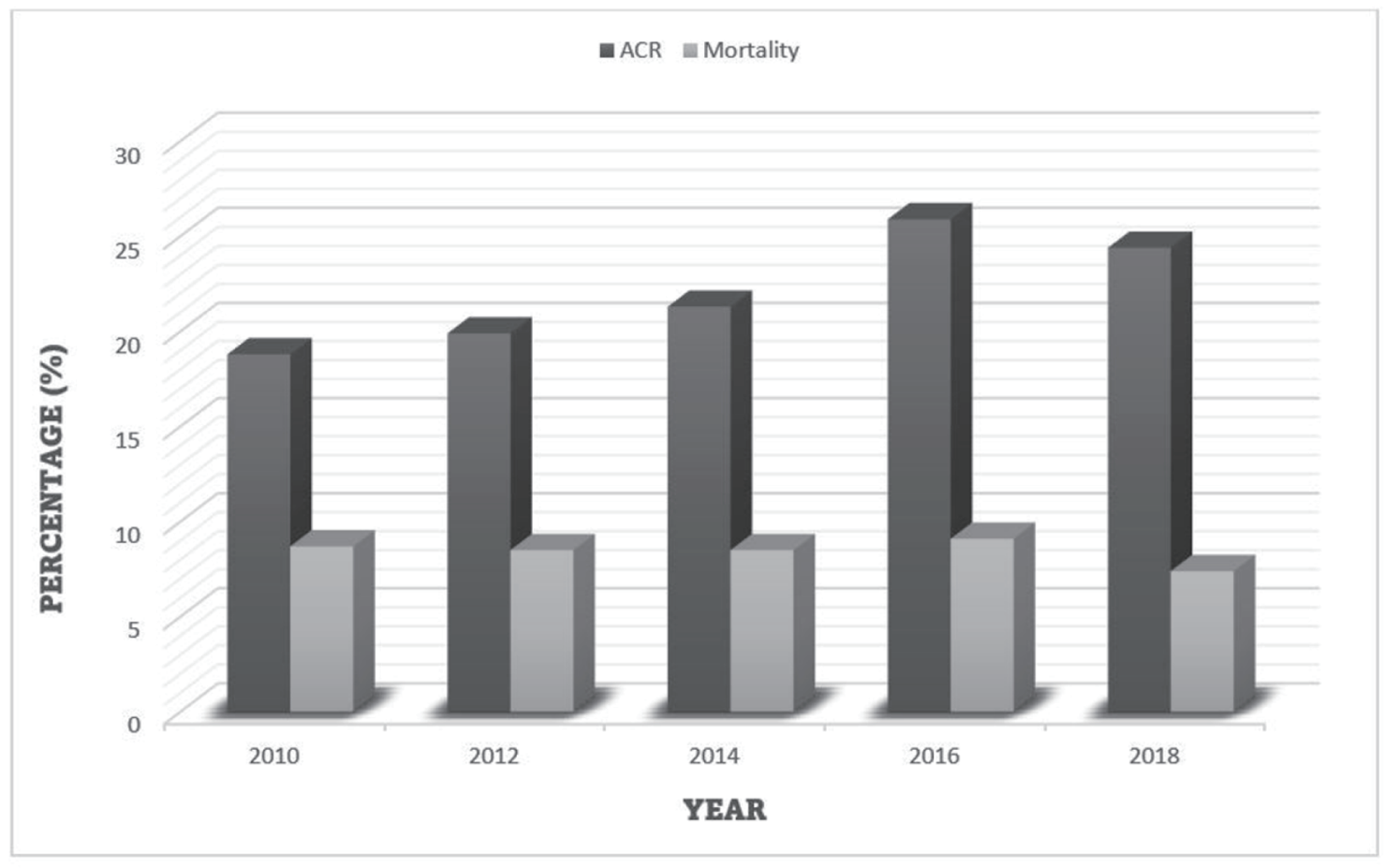

Figure 1. Trend of ACR and inpatient mortality for 30-day readmission of alcoholic hepatitis. ACR: all-cause readmission. 
is expected to rise. A retrospective study conducted from 2010 to 2014 noted a substantial increase in the annual hospital cost to $\$ 164$ million and the total hospitalization days to 22,244 for 30-day readmissions of AH in 2014 [14]. In our study, the mean LOS increased from 6.1 days in 2010 to 6.7 days in 2018 which attributed to an overall increase by $132.5 \%$ from a total 11,275 days in 2010 to 26,220 days in 2018. The mean THC per admission also increased from $\$ 14,285$ in 2010 to $\$ 17,277$ with the total attributable hospital cost increasing by $160.9 \%$ to over $\$ 67$ million by 2018 . From a purely hospital perspective, large bed-size hospitals were found to have the most 30-day readmission of $\mathrm{AH}$ for the study period, followed by medium bed-size and small-bed size hospitals. This may be attributed to the overall higher admission capacity of these larger hospitals. Furthermore, we noted an increasing trend in the proportion of 30-day readmissions of AH from 50.3\% in 2010 to $75.1 \%$ in 2018 for metropolitan teaching hospitals. This may be due to the fact that these metropolitan hospitals are usually located in population dense urban areas and serve as tertiary care referral centers accepting patients from large geographical areas. Additionally, these hospitals are well equipped with a multi-disciplinary team of specialists and the resources required to manage not only $\mathrm{AH}$ readmissions, but also to treat associated complications, if any.

\section{Strength and limitations}

Our study has several strengths and limitations. The key strengths of this study are the study population, the unique study design, and the methodology. The data for this study were gathered from one of the largest, multi-ethnic, publicly available databases in the US developed through a unique Federal-State-Industry partnership. The NRD contains data from hospitals across the US covering about $97 \%$ of the population. Therefore, the results derived from the study are applicable to most hospitals across the US. Additionally, through its unique design and methodology, the study offers a comprehensive analysis of the biogeographic and hospitalizations characteristics along with adverse outcomes associated with 30-day readmissions of $\mathrm{AH}$, adding meaningful information to current literature. Furthermore, the 9-year study periods helped to establish meaningful trends. However, we do acknowledge the limitations associated with this study. The NRD does not contain data on the severity of the disease at the time of readmission or the exact duration after discharge to readmission. Additionally, it does not contain information on the treatment aspects of $\mathrm{AH}$. We were also unable to determine the proportion of patients with associated mood disorders, and those treated in alcohol rehabilitation centers. Moreover, due to the retrospective nature of the study, it is amenable to all biases associated with retrospective studies. Finally, the NRD is an administrative database that uses codes to store information on readmissions; therefore, it is susceptible to coding errors. Despite these limitations, we believe that this study helps us better understand the topic in question and fill the gaps in current knowledge. Through this study we aim to encourage intellectual conversation and promote future research on 30-day readmissions of $\mathrm{AH}$.

\section{Conclusion}

AH is a significant health concern worldwide and in the US. From 2010 to 2018 , there was a rising trend for 30-day readmissions for AH in the US from 1,839 in 2010 to 3,784 in 2018 , which may be due to relapse to alcohol use post hospital discharge or sudden hepatic decompensation in patients with underlying ALD. However, the inpatient mortality rate for these readmissions was on a decline from $8.7 \%$ in 2010 to $7.4 \%$ in 2018 reflecting better access to care and significant improvement in management. There was an increase in the 30day all-cause readmission rate from $18.8 \%$ in 2010 to $24.4 \%$ in 2018, 30-day AH-specific readmission rate from 2.9\% in 2010 to $3.9 \%$ in 2018 , and 30 -day $\mathrm{AH}$ readmission proportion from $18.6 \%$ in 2010 to $20 \%$ in 2018 , but no statistical trend was observed. Additionally, 30-day readmissions of AH places significant burden on the US healthcare system as the total days of hospital stay, and attributable hospital costs increased by $132.5 \%$ and $160.7 \%$, respectively.

\section{Acknowledgments}

None to declare.

\section{Financial Disclosure}

The authors have no financial disclosure.

\section{Conflict of Interest}

The authors report no conflict of interest.

\section{Informed Consent}

As the NRD database lacks patient-specific identifiers, no consent was required for the analysis.

\section{Author Contributions}

Substantial contributions to the conception and design of the work: Dushyant Singh Dahiya and Asim Kichloo. Acquisition, analysis, or interpretation of data for the work: all authors. Literature search and review: all authors. Drafting the work or revising it critically for important intellectual content: all authors. Final approval of the version to be published: all authors. Agreement to be accountable for all aspects of the work: all authors.

\section{Data Availability}

The NRD is a large, multi-ethnic, all-payer publicly available 
inpatient database containing information on more than 18 million hospital stays per year in the US. Its large sample size provides sufficient data for analysis of readmissions for both common and relatively uncommon disorders across all hospital types. The NRD is available at: https://www.hcup-us.ahrq. gov/nrdoverview.jsp.

\section{Abbreviations}

AH: alcoholic hepatitis; AHRQ: Agency for Healthcare Research and Quality; ALD: alcoholic liver disease; CCI: Charlson Comorbidity Index; HCC: hepatocellular carcinoma; HCUP: Healthcare Cost and Utilization Project; ICD: International Classification of Diseases; IRB: Institutional Review Board; LOS: length of stay; NRD: National Readmissions Database; THC: total hospital charge; US: United States

\section{References}

1. Osna NA, Donohue TM, Jr., Kharbanda KK. Alcoholic liver disease: pathogenesis and current management. Alcohol Res. 2017;38(2):147-161.

2. Dguzeh U, Haddad NC, Smith KTS, Johnson JO, Doye AA, Gwathmey JK, Haddad GE. Alcoholism: a multisystemic cellular insult to organs. Int J Environ Res Public Health. 2018;15(6):1083.

3. Basra S, Anand BS. Definition, epidemiology and magnitude of alcoholic hepatitis. World J Hepatol. 2011;3(5):108-113.

4. Sehrawat TS, Liu M, Shah VH. The knowns and unknowns of treatment for alcoholic hepatitis. Lancet Gastroenterol Hepatol. 2020;5(5):494-506.

5. Philips CA, Augustine P, Yerol PK, Rajesh S, Mahadevan P. Severe alcoholic hepatitis: current perspectives. Hepat Med. 2019;11:97-108.

6. Peeraphatdit TB, Kamath PS, Karpyak VM, Davis B, Desai V, Liangpunsakul S, Sanyal A, et al. Alcohol Rehabilitation within 30 days of hospital discharge is associated with reduced readmission, relapse, and death in patients with alcoholic hepatitis. Clin Gastroenterol Hepatol. 2020;18(2):477-485 e475.

7. Healthcare Cost and Utilization Project. Introduction to the HCUP National Inpatient Sample (NIS). The National (nationwide) Inpatient Sample database documentation. Rockville, MD: Agency for Healthcare Research and Quality. Available at: https://www.hcup-us.ahrq.gov/db/ nation/nis/NIS_Introduction_2018.jsp. Accessed September 10, $202 \overline{1}$.

8. Sundararajan V, Quan H, Halfon P, Fushimi K, Luthi JC, Burnand B, Ghali WA, et al. Cross-national comparative performance of three versions of the ICD-10 Charlson index. Med Care. 2007;45(12):1210-1215.

9. Cost-to-Charge Ratio Files. Cost-to-Charge Ratio Files, Rockville, MD: Healthcare Cost and Utilization Project; 2020. Available from: https://www.hcup-us.ahrq.gov/db/ ccr/costtocharge.jsp. Accessed September 10, 2021.

10. Dunn A, Grosse SD, Zuvekas SH. Adjusting health expenditures for inflation: a review of measures for health services research in the United States. Health Serv Res. 2018;53(1):175-196.

11. Louvet A, Labreuche J, Artru F, Bouthors A, Rolland B, Saffers P, Lollivier J, et al. Main drivers of outcome differ between short term and long term in severe alcoholic hepatitis: A prospective study. Hepatology. 2017;66(5):14641473.

12. Altamirano J, Lopez-Pelayo H, Michelena J, Jones PD, Ortega L, Gines P, Caballeria J, et al. Alcohol abstinence in patients surviving an episode of alcoholic hepatitis: Prediction and impact on long-term survival. Hepatology. 2017;66(6):1842-1853.

13. White AM, Slater ME, Ng G, Hingson R, Breslow R. Trends in alcohol-related emergency department visits in the united states: results from the nationwide emergency department sample, 2006 to 2014. Alcohol Clin Exp Res. 2018;42(2):352-359.

14. Adejumo AC, Cholankeril G, Iqbal U, Yoo ER, Boursiquot BC, Concepcion WC, Kim D, et al. Readmission rates and associated outcomes for alcoholic hepatitis: a nationwide cohort study. Dig Dis Sci. 2020;65(4):9901002.

15. Hughes E, Hopkins LJ, Parker R. Survival from alcoholic hepatitis has not improved over time. PLoS One. 2018;13(2):e0192393.

16. Liangpunsakul S, Haber P, McCaughan GW. Alcoholic liver disease in Asia, Europe, and North America. Gastroenterology. 2016;150(8):1786-1797. 